\title{
Comprehensive assessments of germline deletion structural variants reveal the association between prognostic MUC4 and CEP72 deletions and immune response gene expression in colorectal cancer patients
}

Peng-Chan Lin ${ }^{1,2,3,4}$, Hui-O Chen ${ }^{1}$, Chih-Jung Lee ${ }^{1}$, Yu-Min Yeh ${ }^{3,4}$, Meng-Ru Shen ${ }^{5,6,7}$ and Jung-Hsien Chiang ${ }^{1,2^{*}}$

\begin{abstract}
Background: Functional disruptions by large germline genomic structural variants in susceptible genes are known risks for cancer. We used deletion structural variants (DSVs) generated from germline whole-genome sequencing (WGS) and DSV immune-related association tumor microenvironment (TME) to predict cancer risk and prognosis.

Methods: We investigated the contribution of germline DSVs to cancer susceptibility and prognosis by silicon and causal inference models. DSVs in germline WGS data were generated from the blood samples of 192 cancer and 499 non-cancer subjects. Clinical information, including family cancer history (FCH), was obtained from the National Cheng Kung University Hospital and Taiwan Biobank. Ninety-nine colorectal cancer (CRC) patients had immune response gene expression data. We used joint calling tools and an attention-weighted model to build the cancer risk predictive model and identify DSVs in familial cancer. The survival support vector machine (survival-SVM) was used to select prognostic DSVs.

(Continued on next page)
\end{abstract}

\footnotetext{
*Correspondence: jchiang@mail.ncku.edu.tw

'Department of Computer Science and Information Engineering, College of

Electrical Engineering and Computer Science, National Cheng Kung

University, Tainan, Taiwan

${ }^{2}$ Institute of Medical Informatics, National Cheng Kung University, Tainan,

Taiwan

Full list of author information is available at the end of the article
}

(c) The Author(s). 2021 Open Access This article is licensed under a Creative Commons Attribution 4.0 International License, which permits use, sharing, adaptation, distribution and reproduction in any medium or format, as long as you give appropriate credit to the original author(s) and the source, provide a link to the Creative Commons licence, and indicate if changes were made. The images or other third party material in this article are included in the article's Creative Commons. licence, unless indicated otherwise in a credit line to the material. If material is not included in the article's Creative Commons licence and your intended use is not permitted by statutory regulation or exceeds the permitted use, you will need to obtain permission directly from the copyright holder. To view a copy of this licence, visit http://creativecommons.org/licenses/by/4.0/ The Creative Commons Public Domain Dedication waiver (http://creativecommons.org/publicdomain/zero/1.0/) applies to the data made available in this article, unless otherwise stated in a credit line to the data. 
(Continued from previous page)

Results: We identified 671 DSVs that could predict cancer risk. The area under the curve (AUC) of the receiver operating characteristic curve (ROC) of the attention-weighted model was 0.71. The 3 most frequent DSV genes observed in cancer patients were identified as ADCY9, AURKAPS1, and RAB3GAP2 ( $p<0.05)$. The DSVs in SGSM2 and LHFPL3 were relevant to colorectal cancer. We found a higher incidence of FCH in cancer patients than in noncancer subjects $(p<0.05)$. SMYD3 and NKD2DSV genes were associated with cancer patients with $\mathrm{FCH}(p<0.05)$. We identified 65 immune-associated DSV markers for assessing cancer prognosis $(p<0.05)$. The functional protein of MUC4 DSV gene interacted with MAGE1 expression, according to the STRING database. The causal inference model showed that deleting the CEP72 DSV gene affect the recurrence-free survival (RFS) of IFIT1 expression.

Conclusions: We established an explainable attention-weighted model for cancer risk prediction and used the survival-SVM for prognostic stratification by using germline DSVs and immune gene expression datasets. Comprehensive assessments of germline DSVs can predict the cancer risk and clinical outcome of colon cancer patients.

Keywords: Whole-genome sequencing, Cancer risk, Deletion structural variants, MUC4, CEP72

\section{Introduction}

Large-scale germline structural variants, especially deletion structural variants (DSVs), can affect gene expression with a partial or complete loss of gene function and increased cancer risk in patients $[1,2]$. Several studies have reported the germline pathogenic DSVs through whole-genome sequencing (WGS) [3, 4]. For example, patients with germline $R A D 51 C$ exon 5 deletion or $A T M$ exon 9 deletion were confirmed as having hereditary cancer syndrome [5]. Instead of multiplex ligationdependent probe amplification (MLPA) and nextgeneration sequencing (NGS) panels, WGS with multiple cancer-associated DSVs has become more widely used for cancer risk assessment. However, the role of germline DSVs and DSV immune-related association tumor microenvironment (TME) in cancer risk and prognosis had not been sufficiently understood.

To investigate the contribution of germline DSVs to cancer susceptibility and prognosis, we used silicon and causal inference models. Prediction models are important when classifying individuals for predicting the risk and survival stratification to minimize the impact of cancer and optimize treatment [6]. The application of machine learning techniques, such as deep learning (DL) and inherited risk genomic variation analysis, is rapidly developing [7, 8]. As DL has improved the ability to predict inherited cancer genomic susceptibility, we focused on DL as an attention-weighted model with multilayer perceptrons (MLPs) [9], which can reveal the importance of each DSV for predicting cancer risk. Additionally, we used the survival support vector machine (survival-SVM) for selecting the features of prognostic DSVs.

Here we describe the prediction model of germline DSVs in cancer patients with and without family cancer history $(\mathrm{FCH})$. We used a machine learning model for survival stratification to assess the prognosis and demonstrated the biological relevance of germline DSVs and TME-related immune gene expression.

\section{Results}

Germline DSV detection from whole-genome sequencing We applied feature extraction and selection methods to analyze genomics data for the detection of cancerassociated, immune-associated, and prognosis-associated DSVs. (Fig. 1).We utilized the PopDel [10] tool to detect germline DSVs. The WGS data of cancer patients and non-cancer subjects were input simultaneously for joint calling. A total of 14,772 autosomal DSVs with sizes ranging from 500 to 10,000 base pairs were called simultaneously across all samples. We focused our analysis on DSVs occurring in at least $1 \%$ of the samples of both cancer and non-cancer populations at minor allele frequency (MAF) above 5\% [11]. A total of 2919 DSVs that passed the filtering criteria were further used to build a classification model.

\section{Predicting cancer risk with whole-genome DSVs and MLP}

Germline genomic DSVs are known to be associated with increased risk for cancer [12], and several studies have reportedly applied machine learning tools for developing prediction models [13]. To learn the importance of each DSV for classifying cancer or non-cancer samples, we consider the attention-weighted model to be the final approach. Furthermore, the attention-weighted model had the best performance to predict cancer risk. Herein, several machine learning strategies for classification were applied and evaluated. We used an SVM with linear kernel and logistic regression (LR), both of which were well-known linear models. We also used random forests (RF) to test nonlinear results. Moreover, neural network strategies, such as multilayer perceptron and attention-weighted models, were also adopted (Fig. 2a). 


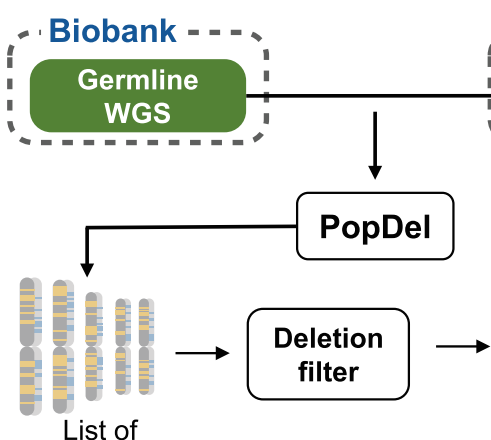

14,772 deletions

671 Deletions

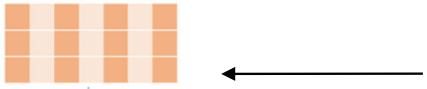

ancer risk-associated deletions table

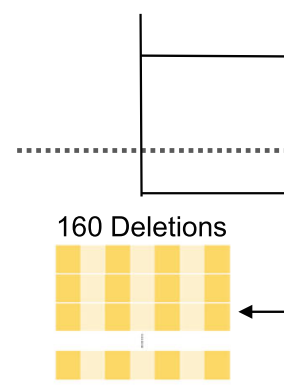

Immune gene expression associated deletion matrix (i)

\section{Study $(\mathrm{N}=192)$ and \\ Reference group \\ $(\mathrm{N}=499)$}

(ii)

\section{DSVs Joint-Calling}

Data preprocessing

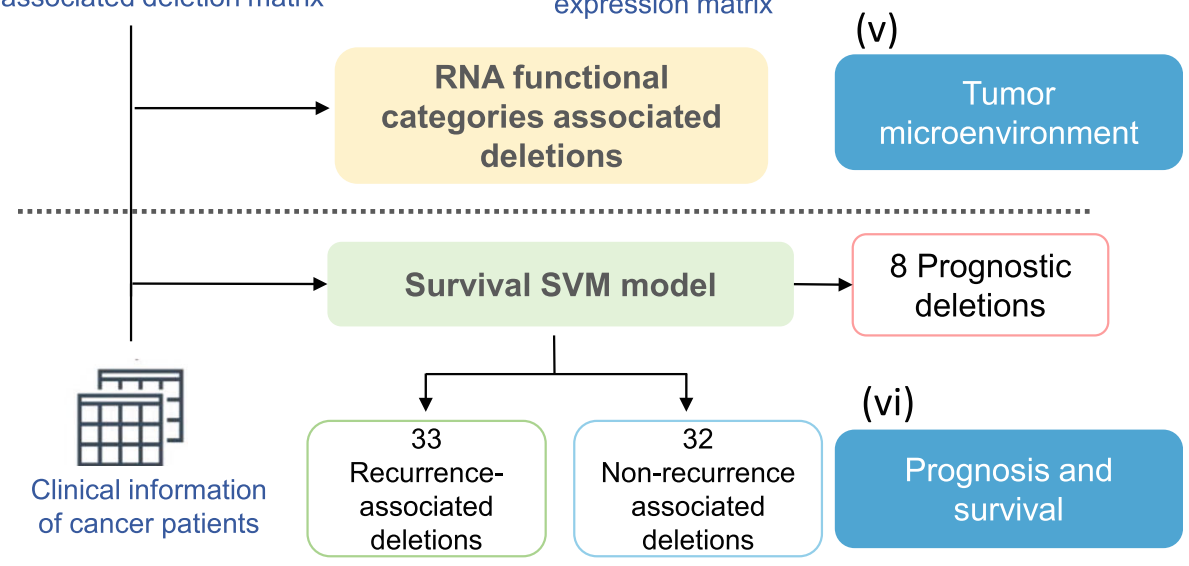

Fig. 1 Study design and workflow. Study design and overall workflow of WGS analysis of germline DSVs and immune gene expression for cancer risk prediction and survival stratification. In total, 192 cancer patients (i) - comprised of 120 with colorectal cancer, 29 with endometrial cancer, 35 with ovarian cancer, and eight with breast cancer-were enrolled in the study group, and 499 non-cancer subjects (i) were included in the reference group. Genomic data, including WGS, gene expression, clinical outcome, and FCH, were collected. First, we used the PopDel method (ii) to detect DSVs and perform data preprocessing (ii) from the WGS analysis of all subjects. The cancer risk predictive model (iii) was built with an attention-weighted model. We also studied DSVs in familial cancer (iv). Second, we examined the relationship between DSVs and the tumor microenvironment $(v)$. Immune gene expression data were normalized. We constructed an immune gene expression-associated DSV correlation matrix with the point-biserial correlation. Third, a machine learning method with a survival support vector machine (survival-SVM) and KaplanMeier survival analysis was applied to examine prognosis and survival (vi) 

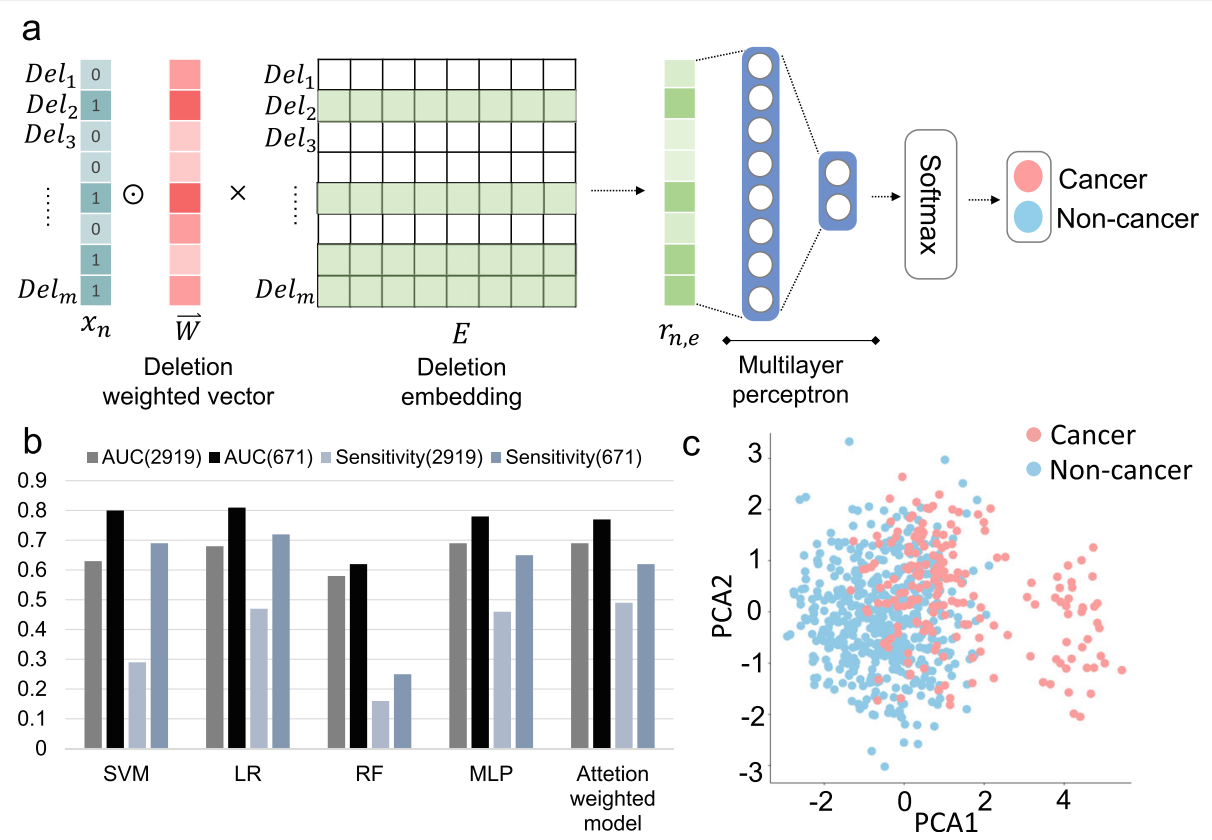

Fig. 2 Feature selection of DSVs to distinguish cancer and non-cancer subjects. a The architecture of the attention-weighted model for selecting the cancer risk DSV features. The primary purpose was to classify cancer or non-cancer subjects by the neural network. This was a MLP model based on the attention mechanism. We used $n$ samples $\left(x_{n}\right)$ as input in the attention-weighted model: every sample had $m\left(D e l_{m}\right)$ filtered deletions. A value of 1 in the deletion vector indicates that the sample has the specific deletion, while 0 implies no deletion. A weighted vector ( $\rightarrow W$ ) is associated to the input layer to identify the importance of each deletion (red color gamut). Additionally, an embedding layer (E represents the embedding table, e denotes the embedding size) is applied to reduce the feature size and each deletion. We took the sum of each column and obtained a vector that can represent the information of the input deletion features $\left(r_{n, e}\right)$; this is the input of multilayer perceptron. The output of MLP utilizes the SoftMax layer. The output labels are cancer or non-cancer subjects. $\mathbf{b}$ Performance of five machine learning strategies (attention-weighted model, MLP SVM, RF, and LR) for cancer risk prediction with different number of features (2919 and 671 cancer-associated DSVs). The attention-weighted model was more sensitive (AUC $=0.71$, sensitivity $=0.57$ ) than the other methods. All of the models performance are improved with 671 cancer-associated DSVs. c PCA plot by cancer-associated DSVs. Red dots represent cancer subjects, and blue dots represent non-cancer subjects. A total of 671 cancer-associated DSVs with positive weights were used for PCA. DSVs can distinguish cancer and non-cancer subjects

The area under the curve (AUC) from the receiver operating characteristic curve (ROC) and the performance of models (i.e., sensitivity) are crucial for clinical use. Among these methods, the attention-weighted model $($ AUC $=0.71$, sensitivity $=0.58)$ performed the best with 2919 DSVs (Fig. 2b and Supplementary Fig. 1). All of the model's performances were improved with 671 cancerassociated DSVs. In total, 671 of 2919 significant DSVs were selected for the prediction of cancer risk with positive weights from the attention-weighted model (Supplementary Table 1A). There were no demographic biases in the population data (Supplementary Fig. 2). The size and distribution of deletions on each chromosome were no different between cancer patients and non-cancer subjects (Supplementary Fig. 3). The cancer and noncancer samples could be distinguished with 671 DSVs in principal components analysis (PCA). The 671 DSVs were divided into two clusters by using hierarchical clustering. We used REACTOME [14] to perform pathway enrichment of genes. There were 92 genes in the first cluster and 125 genes in the second cluster. In the first cluster, there were 11 genes (MUC17, MUC19, MUC4, MUC6, GALNT9, B3GNTL1, KCNMB2, UNC13B, RIMS1, SMYD3, MYT1) that enriched 19 significant pathways ( $p$ value $<0.05)$ which were relevant to $\mathrm{O}$ linked glycosylation of mucins, Neurotransmitter Release Cycle, defective factor causing familial hyperphosphatemic tumoral calcinosis, etc. In the second cluster, there were 27 genes (KCNJ6, ADCY9, GNG2, ADCY8, PFKFB3, ESR1, MUC12, MUC4, GALNT9, B3GNTL1, ATP13A4, ATP2B4, ATP11A, SBF2, ADAP1, F11, CACN G7, KCNIP1, CACNA1C, RPTOR, PSMA8, ACTN2, PPP6R2, CYP4F11, CYP4F12, SOD2) that enriched 47 significant pathways $(p$ value $<0.05)$ which involved defective factor causing hereditary angioedema, Ion transport by P-type ATPases, diseases of hemostasis, etc. (Supplementary Table 2). However, there were no differences between each type of cancer (Supplementary Fig. $4 \mathrm{~A})$. Further analyses were conducted to determine the genes with DSVs and relevant pathways related to different cancer types. The result indicated that the DSVs in SNTG2, PCMT1, DACT2, CBX3, ATP11A, and SHC2 
were associated with breast cancer. DSVs in SGSM2 and $L H F P L 3$ were relevant to colorectal cancer, whereas $A D A P 1, D L G A P 2, E R C 1$, and PPP6R2 were related to gynecologic cancer (Supplementary Fig. 4B and C).

\section{The mutational landscape of DSVs and their significance in familial cancer patients}

The germline mutational landscape of DSVs plays an important role in cancer patients with or without FCH. Patients who have one or more blood relatives within third-degree suffering from any types of cancer are considered having family cancer history. The odds ratios were estimated to identify which genes with DSVs were associated with FCH. We chose the top 10 DSV genes associated with an increased risk of cancer (odds ratios $(\mathrm{OD})>1$ ) and the bottom 10 DSV genes associated with a decreased risk of cancer $(\mathrm{OD}<1)$ from 671 DSVs associated with cancer risk
(Fig. 3a). The top 10 genes frequently observed in cancer patients were ADCY9, RAB3GAP2, AURKAPS1, EYS, SHC2, DPP6, FREM2, ESR1, TBC1D22A, and $A C T N 2$. The ten genes frequently observed in noncancer subjects were SNTG2, LHFPL3, DACT2, NKAI N2, KALRN, ABR, LMNTD1, PLEKHA7, DOC2B, and ADPRHL1 (Supplementary Table 3). We also studied the prevalence and spectrum of well-known germline cancer susceptibility genes in our subjects [15]. The frequencies of 26 cancer susceptibility genes are shown in Fig. 3b. Deletions in the FANCA, POLD1, and STK11 genes were observed in cancer patients only. The frequency of gene deletions was almost the same between cancer and non-cancer subjects. The mutational landscape of DSV genes is shown in Fig. 3c. There were 57 cancer-associated DSV genes with a $p$ value $<0.05$ in the cancer and non-cancer groups (Supplementary Table 4).

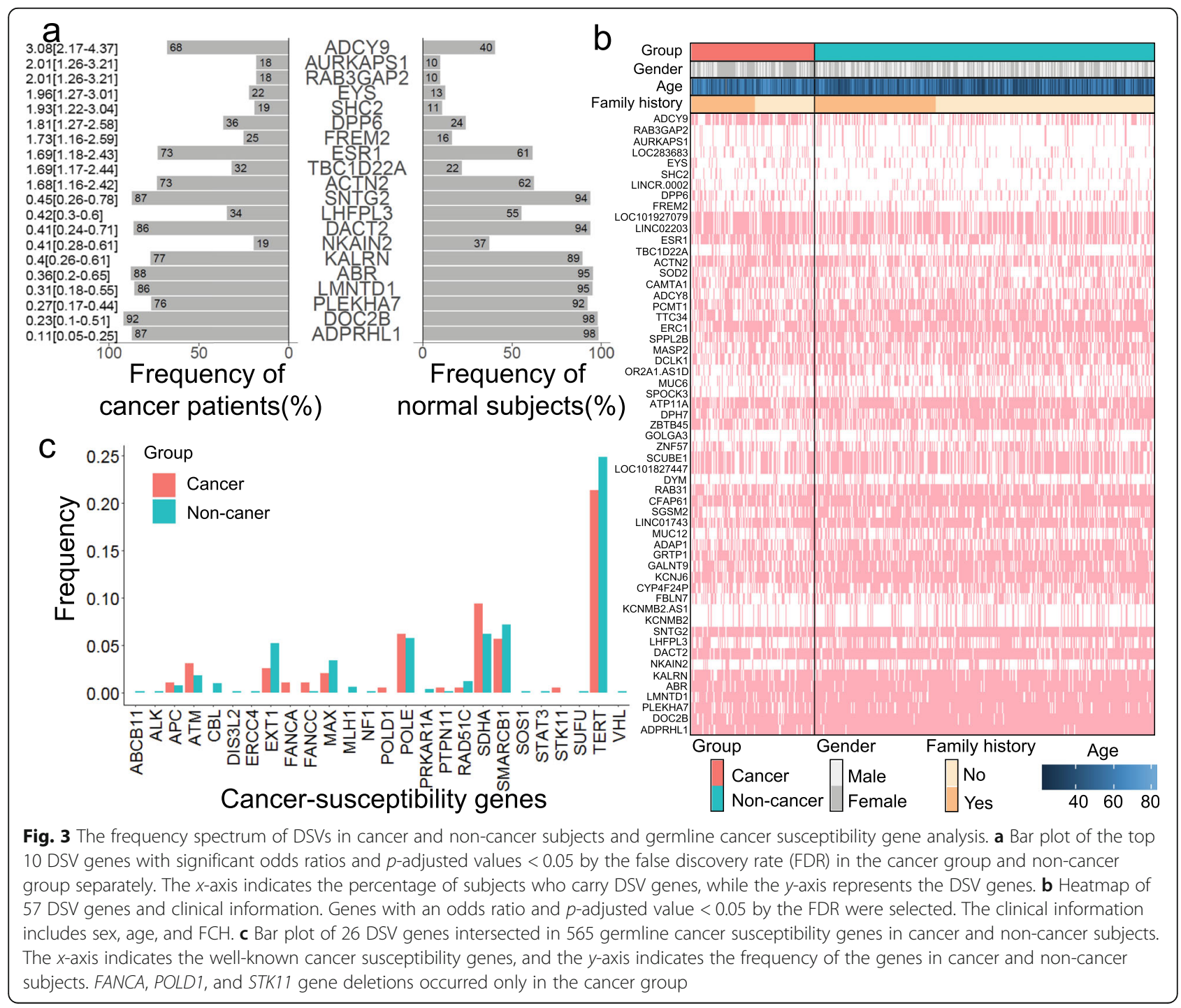


In this study, we found a higher incidence of $\mathrm{FCH}$ in cancer patients than in non-cancer subjects (Fig. $4 \mathrm{a}, p$ value $=0.0003)$. The study had included 177 cancer patients and 407 non-cancer subjects with family history. Ninety-one of 177 cancer patients presented with family cancer history. One hundred seventy-seven of 407 noncancer subjects presented with family cancer history. The relationship of cancer and non-cancer subjects with family cancer history was tested by Fisher's exact test. A higher percentage of cancer patients have a family cancer history (Fig. 4a). The five most cancer types in family-affected members are liver cancer, colorectal cancer, lung cancer, breast cancer, and gastric cancer (Supplementary Table 5).

Moreover, certain DSV genes were associated with cancer or non-cancer subjects with or without FCH (Fig. 4b and c). MGAT4C, HSPA4L, ZSCAN5A, LOC100505841, and $N A L C N$ gene deletions were associated with cancer patients without FCH $(p<0.05)$, while SMYD3 and $N K D 2 D S V$ genes were associated with cancer patients with FCH $(p<0.05)$. HHIPL2, XPO1, SALRNA1, ZBTB45, ANP32AP1, ACTR3BP5, LOC100129138, GPR45, and $C A B 39 L$ gene deletions were associated with non-cancer subjects without FCH $(p<0.05)$, while $R A B 9 B P 1$, $L O C 101928523$, and MALRD1 gene deletions were related to non-cancer patients with FCH $(p<0.05)$. Consequently, we inferred that subjects with $\mathrm{FCH}$ carrying SMYD3 or $N K D 2$ gene deletions may have a higher cancer incidence. As illustrated in Fig. 4d, the volcano plot shows eight significant DSV genes based on the Cox's proportional hazards model for survival analysis (Supplementary Table 6).

\section{The clinical impact of immune gene expression-related DSVs in colorectal cancer patients}

The host immune system differentially participates in the tumor microenvironment. Cancer often develops because of the immune system disturbance caused and functional disorder. The germline DSVs influence aberrant gene expression in tumors [16]. Therefore, we studied the functions associated with 160 immune gene expression-associated DSVs with correlation coefficients of $>0.3$, which were selected based on the point-biserial correlation to understand the clinical impact of their deletions (Supplementary Table 1B). There are six categories of immune gene functions: housekeeping, checkpoint pathways, cytokine signaling, lymphocyte markers, lymphocyte regulation, and tumor characterization. A total of 57 DSV genes were correlated with the six functional immune response categories; the PTPRN2 gene

\begin{tabular}{|c|c|c|c|c|}
\hline \multirow{2}{*}{\multicolumn{2}{|c|}{ a }} & \multicolumn{2}{|c|}{ Cancer } & \multirow[b]{2}{*}{ Total } \\
\hline & & $(+)$ & $(-)$ & \\
\hline \multirow{4}{*}{$\begin{array}{l}\text { Family } \\
\text { cancer } \\
\text { history }\end{array}$} & $(+)$ & 91 & 177 & 268 \\
\hline & $(-)$ & 86 & 317 & 403 \\
\hline & Total & 177 & 494 & 671 \\
\hline & & $\begin{array}{l}\text { her's } \\
\text { (OR } \\
\text { alue }\end{array}$ & & \\
\hline
\end{tabular}

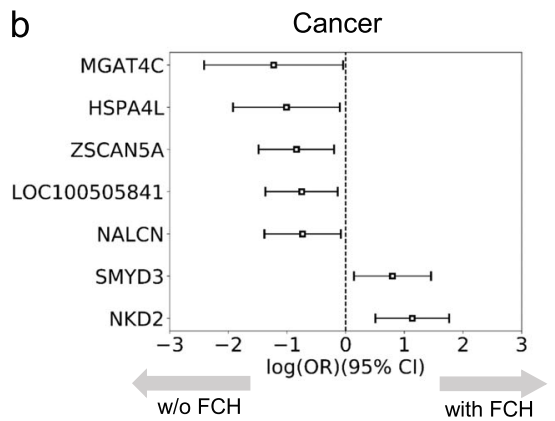

c
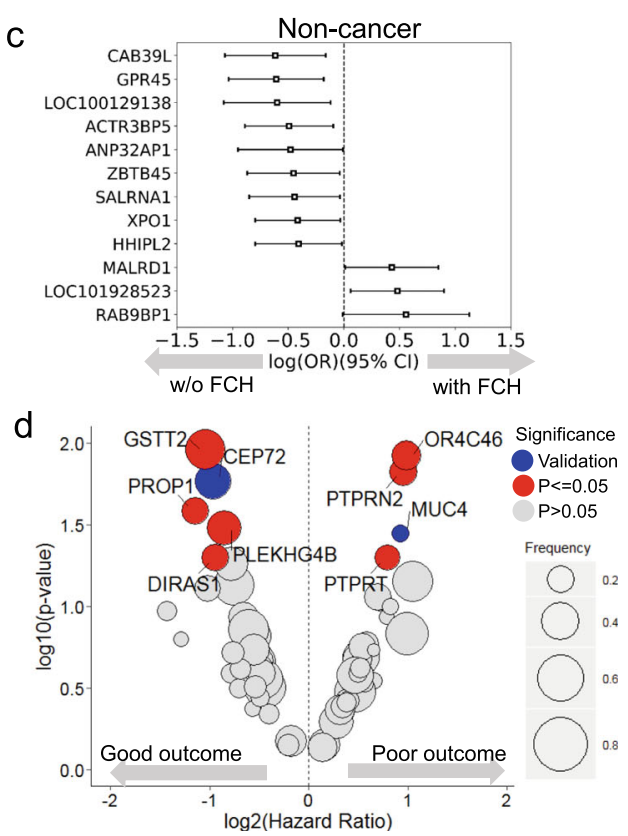

Fig. 4 DSV genes and survival analysis in cancer patients with or without family cancer history. a Table of the association between cancer and $\mathrm{FCH}$. The subjects who had FCH had a higher risk of developing cancer 1.89 [1.33-2.68] than the subjects without FCH (Fisher's exact test $p=$ 0.0003). The family cancer history is related to first- and second-degree relatives of patients with any cancer. $\mathbf{b}$ Fisher's exact test and odds ratio were applied to measure the relationship between each DSV gene and FCH. Forest plot of cancer patients with and without FCH. The DSV genes are SMYD3 and NKD2 in cancer patients with FCH. The DSV genes are MGAT4C, HSPA4L, ZSCAN5A, LOC100505841, and NALCN in cancer patients without FCH. c Forest plot of non-cancer subjects with and without FCH. The DSV genes are MALRD1, LOC101928523, and RAB9BP1 in non-cancer subjects with FCH. There are nine DSV genes in non-cancer subjects without FCH. d Point plot of the log2 hazard ratio DSV genes and log $10(p$ value). The size of the point indicates the frequency of the DSV gene in cancer subjects, and the red marks indicate the eight DSV genes with a $p$ value $<0.05$. Blue points (MUC4 and CEP72 gene deletions) show the validated results 
deletion had the highest frequency (Fig. 5a and Supplementary Table 7). STNG2 and LOC105376360 gene deletions (Fig. 5a) were related to lymphocyte regulation and housekeeping ( $p$-adjusted value less than 0.05), while CEP72 and ZZEF1 gene deletions had high occurrences in the housekeeping and cytokine signaling categories, respectively (Fig. 5a).

We selected 65 prognosis-associated DSVs by using survival support vector machine (survival-SVM) [17], which had the highest predicted score for survival-SVM. We used 65 prognosis-associated DSVs among 160 immuneassociated DSV genes and constructed a heatmap (Fig. 5b and Supplementary Table 1C). These prognosis-associated DSVs were grouped into poor (33 recurrence-associated DSVs) and better (32 non-recurrence-associated DSVs) prognostic groups using Cox's proportional hazards model. There were more poor prognostic deletions in the tumor characterization functional category (e.g., $M U C 4$ and PTPRN2 gene deletions) and better prognostic deletions in the lymphocyte regulation functional category (Fig. 5b). We then stratified the patients into two groups by prognostic deletions that have different clinical outcomes. Group 1 (G1) was the patient who has more recurrence-associated DSVs than non-recurrenceassociated DSVs. According to the Kaplan-Meier curve, these patients in G1 experienced a poor clinical outcome $(p<0.05)$ (Fig. 5c). Patients in group 2 (G2) had better outcomes whose non-recurrence-associated DSVs are more than recurrence-associated DSVs.

\section{The biological relevance of germline DSVs and tumor microenvironment immune genes}

The tumor microenvironment can affect prognosis and shape therapeutic resistance [18]. Overexpression of the

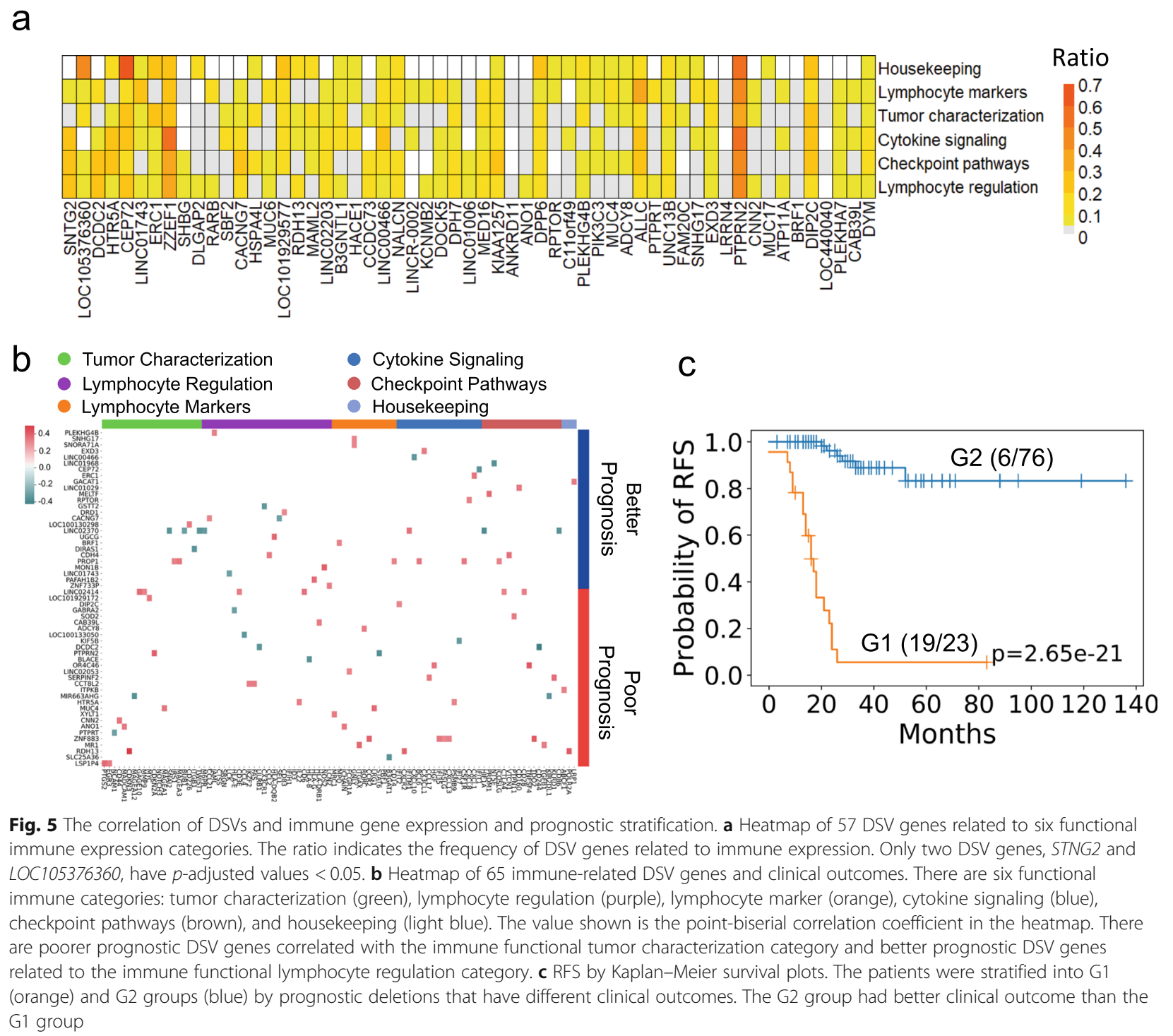


immune MAGEA1 gene, a member of the MAGEA gene family, in tumor and stromal cells is associated with a poor prognosis and an ideal candidate for tumor immunotherapy $[19,20]$. MAGE1 was highly expressed in a previous study on colorectal cancer [20]. In our data, we showed that colorectal cancer patients with germline MUC4 gene deletion experienced a poor clinical outcome (Fig. 6a). Seven of 13 patients with a germline $M U C 4$ gene deletion experienced recurrence. Moreover, the MUC4 gene deletion was positively correlated with $M A G E 1$ expression, which indicated that SV deletion resulted in increased MAGE1 expression (Fig. 6a). With the use of the STRING database [21], we also demonstrated protein-protein interactions between the transmembrane mucin family, including MUC4 and MAGE1
(Fig. 6b). The functional protein association networks indicated that the MUC4 gene deletion might influence the expression of MAGE1.We hypothesized that germline DSVs could affect immune MAGEA1 expression and correlate with a poor prognosis.

Here, we also showed that eight prognostic DSVs can affect RFS by expressing tumor microenvironment immune genes. In our cohort, the eight prognostic deletions were correlated with immune gene expression and survival in colorectal cancer stage III patients (Supplementary Fig. 5 and Supplementary Fig. 6). To understand the cause-effect relationship of this result, we applied causal modeling and implemented the PC algorithm by $\mathrm{R}$ package CompareCausalNetworks [22]. The $\mathrm{PC}$ algorithm uses conditional independence tests for

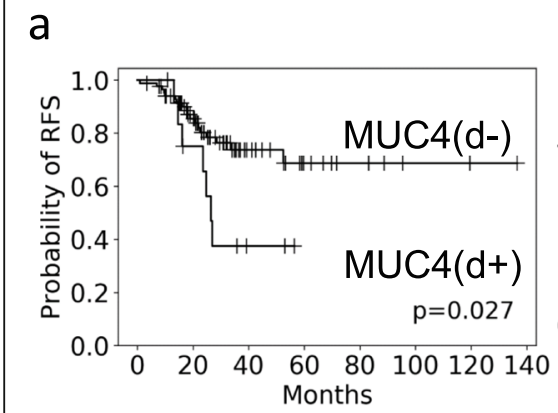

b
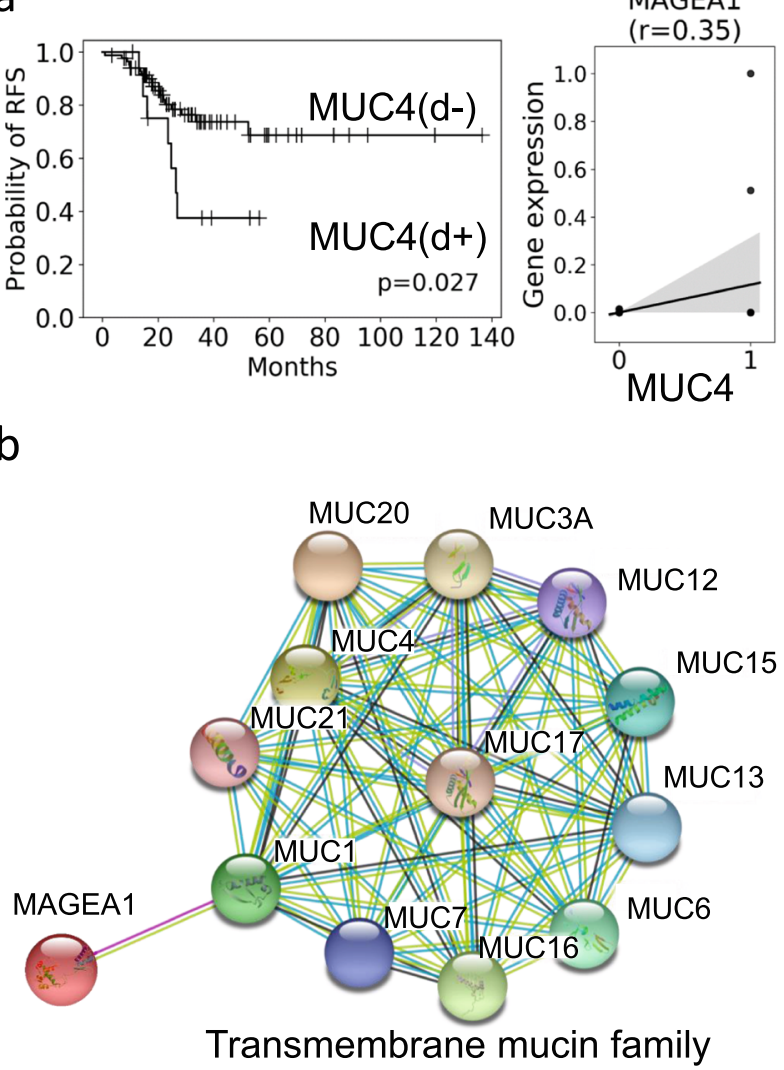

C

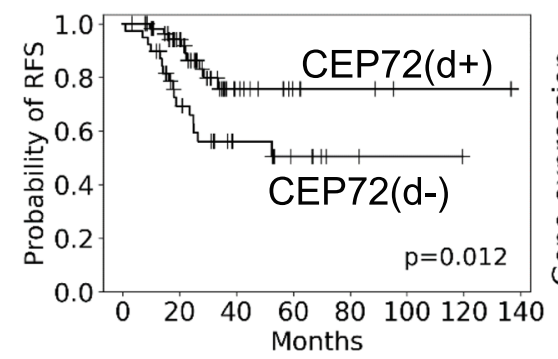

d

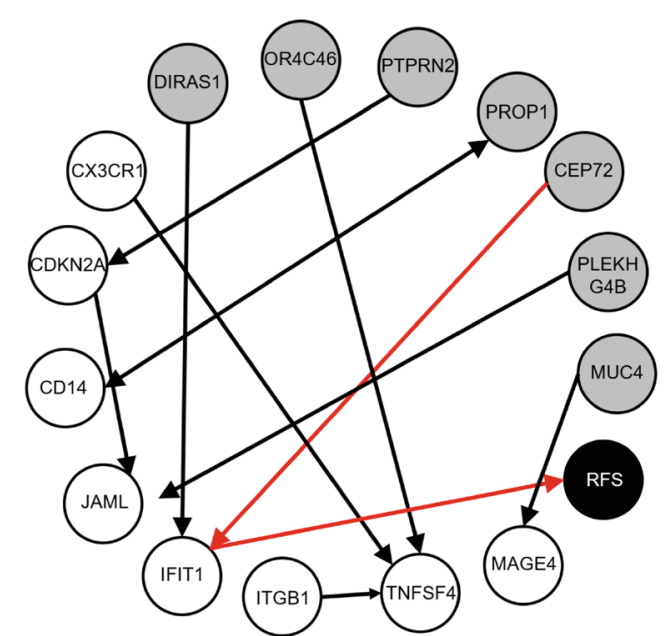

Fig. 6 Protein-protein interactions and the causal inference model. a Kaplan-Meier survival plot of the MUC4 gene DSVs. MUC4 (d-) indicates that cancer patients have no MUC4 gene deletion. MUC4 $(d+)$ indicates that cancer patients have the MUC4 gene deletion. RFS indicates recurrence-free survival. The survival analysis showed that patients with MUC4 (d-) had a better clinical outcome ( $p=0.027)$, and colorectal cancer patients with the MUC4 gene deletion were associated with increased immune gene (MAGE1) expression. The right column shows the MUC4 gene deletion and MAGEA1 expression correlation plot $(r=0.35)$. b The STRING database was used to show protein-protein interactions of the transmembrane mucin family, including MUC1, MUC4, and MAGEA1. c Kaplan-Meier survival plot of CEP72 DSV. CEP72 (d-) indicates that cancer patients have no CEP72 gene deletion. CEP72 $(d+)$ indicates that cancer patients have a CEP72 gene deletion. RFS indicates recurrence-free survival. The survival analysis showed that patients with CEP72 $(\mathrm{d}+)$ had a better clinical outcome $(p=0.012)$, and patients with the CEP72 gene deletion were associated with decreased immune gene (IFIT1) expression. The left figure shows the CEP72 gene deletion and IFIT1 expression correlation plot $(r=0.36)$. d Causal inference model of DSVs, immune gene expression, and RFS. Gray circles represent DSV genes, white circles represent immune expression genes, and the black circle represents RFS. The arrow indicates the causal effect pair. The red arrow pair indicates the RFS causal inference-associated pairs. The causal inference model showed that CEP72 could affect RFS by IFIT1 
model selection in graphical modeling with directed acyclic graphs [23]. Our results showed that deletion of the oncogene CEP72 could affect RFS by IFIT1 immune expression. IFIT1 is an abundant product of interferonstimulating genes that correlates with a poor prognosis in cancer [24].

In this study, we demonstrated the possible biological relevance of the MUC4 gene deletion and MAGE1 expression and found the causal relationships among CEP72 gene deletion, IFIT1, and RFS (Fig. 6d). MUC4 is a transmembrane mucin family member, which is expressed in airway epithelial cells and body fluids. MUC4 plays an important role as a potential candidate for diagnostic and treatment in cancer [25]. CEP72 is the critical protein for the structural integrity of the centrosome and maintaining microtubule-organizing activity [26]. These results indicate that germline DSVs might affect prognosis by expressing tumor microenvironment immune genes.

\section{Discussion}

Advances in machine learning technologies have led to the use of deep learning prediction models for cancer prevention. Here, we applied WGS of germline DSVs for predicting cancer risk and machine learning methods for assessing immune-related prognosis. Our results highlighted the following: (i) a cancer risk predictive model was established with 671 DSVs and an attentionweighted neural network, (ii) potential markers for inherited cancer risk were identified in cancer patients with or without FCH, (iii) 57 DSVs were correlated with six immune functional categories, (iv) 65 prognostic deletions were identified in order to construct a survival model for clinical outcome stratification, and (v) the possible mechanisms and biological relevance of 2 germline deletions in the expression of two immune genes were presented. Germline WGS and immune gene expression profiling are excellent tools for predicting cancer and stratifying prognosis in colorectal cancer patients.

Traditionally, a small subset of gene alteration features that could predict and classify types of cancer were selected by different machine learning models [27]. However, gene-gene interactions can significantly complicate the search for disease-associated genes. Genes play various essential roles in cancer biology, and each gene carries a different weight importance in the clinical outcome. Deep learning can employ an automatic weight learning feature that can allow complex predictions. In this study, we built a deep learning classification model to identify unique biological features that can differentiate between cancer and non-cancer subjects. Using population-based designs, we identified 671 DSVs associated with the risk of cancer. We found that PCA could distinguish between cancer and non-cancer subjects using these 671 DSVs.

We found that the deletion occur in the LHFPL3 gene, which is relevant to colorectal cancer. The DSV located at chromosome 7 starts with 104,473,711 end with 104, 474,263; the length of DSV was 552 bps. The study has found that LHFPL3, the expression of miR-218-5p and miR-138-5p, was downregulated, which correlates to a reduction in cell activity, proliferation, and invasive human ability glioma cells [28]. The deletion in LHFPL3 leads to gene loss of function, which caused a worsening prognosis in colorectal cancer patients. The DSV in $C B X 3$ is located at chromosome 7 , and the region starts with 26,241,421 ends with 26,245,980. The total length of DSV was $4559 \mathrm{bps}$ (Supplementary Table 1). The result indicated that the deletion in $C B X 3$ was associated with breast cancer. The Chromobox (CBX) family proteins have epigenetic regulatory function and transcriptionally repress target genes through chromatin modification. The mRNA expression of $C B X 3$ has been found to affect the outcome of breast cancer in different subtypes. $C B X 3$ mRNA high expression was correlated to worsening RFS for all breast cancer patients [29].

Many hereditary cancer syndromes have now been defined and attributed to specific germline-inherited mutations. Cancer development is related to accumulating genetic alterations. In this study, we studied the evolution pattern of DSVs in cancer patients with or without $\mathrm{FCH}$. We found that subjects with FCH had a higher incidence of developing cancer and may have initially inherited three DSV genes, namely, MALRD1, LOC101928523, and RAB9BP1. They developed cancer after acquiring two DSV genes: NKD2 and SMYD3. However, patients without $\mathrm{FCH}$ may have a different evolution pattern of DSVs. Initially, they inherited nine DSV genes-CAB39L, GPR45, LOC1001291138, ACTR3BP5, ANP32AP1, ZBTB45, SALRNA1, XPO1, and HHIPL2-and developed cancer after acquiring five DSV genes-MGAT4A, HSPA4L, ZSCAN5A, LOC100505841, and $N A L C N$. We focused on eight signaling pathways associated with the aforementioned DSV genes [30]. The most significant pathway enriched with DSV genes for subjects with FCH was metabolic regulation while for subjects without FCH was transport regulation. These results imply that subjects with or without $\mathrm{FCH}$ may develop cancer through different signaling pathways. These DSVs may become useful screening markers.

The result from each classification was the average after five-fold cross validation. The 192 cancer patients and 499 non-cancer samples data were divided into a training set and testing set. We randomly chose $80 \%$ samples as the training data and $20 \%$ samples as the testing set in each fold. Because the DSV analysis was started with BAM file and lack of 
samples, there was no other public data can be used as validation data.

Genetic alterations from nature vs nurture: What determines cancer risk and prognosis? We hypothesized that germline DSVs mold the tumor microenvironment and immune gene expression, impacting the clinical outcome. In this study, we wanted to examine the correlation of germline deletions and immune response genes to understand the potential mechanisms by which the tumor microenvironment can affect clinical outcomes [31]. We classified germline structural deletions by the expression of tumor microenvironment-based immune responseassociated genes. There were significantly poorer prognostic deletions in the tumor characterization category and better prognostic deletions in the lymphocyte regulation category. Eight prognostic deletions associated with immune gene expression were identified, including $H G F$, $C D K N 2 A$, and ITGB1. They were also reported as poor prognostic factors in a previous study [32].

Beyond the traditional signaling factor statistical survival model, we used the survival-SVM and Cox's proportional hazards model to select 65 prognostic deletions. We proposed a method to classify risk and non-risk groups by prognostic deletions and identified 57 prognostic DSVs as possible markers for survival stratification and prognosis assessment. From the bioinformatics database and casual inference model, we also demonstrated that immune-associated gene expression may influence the clinical outcome of some germline deletions. The possible mechanism which affects tumor microenvironment survival was shown, but further molecular validation is needed.

\section{Conclusions}

In conclusion, we used genomic data, including WGS and immune gene expression data, and two explainable machine learning models to establish cancer risk predictive models and a prognosis assessment tool that could be useful for cancer prevention and potential therapeutic strategies. Moreover, we need further functional studies to know the biological information of the DSV genes.

\section{Materials and methods}

\section{Enrollment of cancer patients and non-cancer healthy} subjects

This is a hospital-based cohort study of cancer patients. Eligible cancer patients were age $\geq 20$ years with histologically confirmed pathological stage II-III adenocarcinoma of colon or rectum, stage II-IV endometrial cancer, I-IV epithelial ovarian cancer, or I-IV breast cancer, an Eastern Cooperative Oncology Group performance status (ECOG PS) of $0-1$, and adequate organ function. Patients are willing to provide blood samples for research purposes and written informed consent. Exclusion criteria were receiving chemotherapy within 6 months, other malignancies, and life expectancy less than 1 year. Clinical information, including detailed cancer family history and blood sampling for WGS, health, and lifestyle data of 499 non-cancer normal Taiwanese people ages 30-70, were obtained from Taiwan Biobank.

A total of 192 cancer patients, including eight with breast cancer, 120 with colorectal cancer, 29 with endometrial cancer, and 35 with ovarian cancer, were recruited for the study at the NCKUH between January 2015 and January 2017. Follow-up continued through October 2018. Clinical information (detailed family cancer history (FCH)), tissue, and blood samples for DNA extraction and WGS were collected at the time of enrollment. The NCKUH institutional review board approved this study (A-ER-103-395 and A-ER-104-153), and all participants provided written informed consent. WGS, health, and lifestyle data of 499 non-cancer Taiwanese people were obtained from the Taiwan Biobank as reference (Fig. 1). Of all 99 CRC patients, the distribution of gender was almost the same. The median age of these patients was 58 years. The prevalent primary tumor site was the left colon (80.8\%). Family cancer history is related to first- and second-degree relatives of patients with any cancer. Family cancer history and recurrence were not significantly different. There was no significant difference between recurrence and tumor characteristics, such as tumor site, tumor invasion stage $(\mathrm{T})$, or nodal stage $(\mathrm{N})$. In the genetic features of colorectal cancers, there was no significant difference between recurrence and Mismatch Repair (MMR), KRAS, and TP53 status. There were no significant in clinic pathological differentiation and histology features (Table 1).

\section{Study design and workflow}

To develop the risk and prognostic stratification model, we collected genomic and clinical information, including $\mathrm{FCH}$, such as survival and $\mathrm{FCH}$, from 192 cancer patients at National Cheng Kung University Hospital (NCKUH) and 499 normal subjects without cancer in the Taiwan Biobank [33] with four aims. First, we aimed to build the cancer risk prediction model with germline DSVs. Second, we studied the spectrum and frequency of DSV genes in cancer patients with or without FCH. Third, we aimed to observe whether genes with DSVs would impact the tumor microenvironment's immune response gene expression. Fourth, we stratified the cancer patients' clinical outcomes by immune-related DSVs and investigated the DSVs' relationship and biological relevance. Figure 1 shows the overall workflow of this study. 
Table 199 CRC patients characteristic

\begin{tabular}{|c|c|c|c|}
\hline Characteristic & $\begin{array}{l}\text { Recurrence } \\
(N=25)\end{array}$ & $\begin{array}{l}\text { Non-recurrence } \\
(N=74)\end{array}$ & $P$ value \\
\hline Age & & & 0.606 \\
\hline$<65$ & 17 (68\%) & $55(74.3 \%)$ & \\
\hline$\geq 65$ & $8(32 \%)$ & $19(25.7 \%)$ & \\
\hline Gender & & & 0.645 \\
\hline Male & $11(44 \%)$ & $38(51.4 \%)$ & \\
\hline Female & $14(56 \%)$ & $36(48.6 \%)$ & \\
\hline Tumor location & & & 0.576 \\
\hline Left & $21(84 \%)$ & $55(74.3 \%)$ & \\
\hline Right & $4(16 \%)$ & $17(18.9 \%))$ & \\
\hline Differentiation & & & 0.527 \\
\hline Well & $3(12 \%)$ & $4(5.4 \%)$ & \\
\hline Moderate & $21(84 \%)$ & $66(89.2 \%)$ & \\
\hline Poor & $1(4 \%)$ & $4(5.4 \%)$ & \\
\hline Adenocarcinoma & & & 0.707 \\
\hline Mucinous & $2(8 \%)$ & $10(13.5 \%)$ & \\
\hline Nonmucinous & $23(92 \%)$ & $64(86.5 \%)$ & \\
\hline Tumor invasion stage & & & 0.733 \\
\hline $\mathrm{T} 1 / \mathrm{T} 2$ & $4(16 \%)$ & $9(12.2 \%)$ & \\
\hline $\mathrm{T} 3 / \mathrm{T} 4$ & $21(84 \%)$ & 65 (87.8\%) & \\
\hline Tumor nodal stage & & & 0.292 \\
\hline No/N1 & $16(64 \%)$ & $57(77 \%)$ & \\
\hline $\mathrm{N} 2$ & $9(36 \%)$ & $17(23 \%)$ & \\
\hline Family cancer history & & & $>0.99$ \\
\hline Yes & $11(44 \%)$ & $32(43.2 \%)$ & \\
\hline No & $14(56 \%)$ & $42(56.8 \%)$ & \\
\hline Mismatch repair status & & & 0.325 \\
\hline Proficient & 25 (100\%) & 68 (91.9\%) & \\
\hline Deficient & $0(0 \%)$ & $6(8.1 \%)$ & \\
\hline KRAS status & & & 0.3245 \\
\hline Mutated & $12(48 \%)$ & 25 (34.2\%) & \\
\hline Wild & $13(52 \%)$ & $48(65.8 \%)$ & \\
\hline TP53 status & & & $>0.99$ \\
\hline Mutated & $22(88 \%)$ & $64(88 \%)$ & \\
\hline Wild & $3(12 \%)$ & $9(88 \%)$ & \\
\hline
\end{tabular}

\section{Germline WGS}

Genomic DNA from collected blood samples was quantified with a Qubit fluorescence assay (Thermo Fisher Scientific) and sheared with an S2 instrument (Covaris). Library preparation was carried out using the TruSeq DNA PCR-Free HT Kit (Illumina). Individual DNA libraries were measured with 2100 Bioanalyzer (Agilent) qPCR and Qubit (Thermo Fisher Scientific). All flow cells were sequenced on a HiSeq 2500 sequencer (Illumina) using SBS kit V4 chemistry (Illumina). FastQC was used to check read quality, and the resulting reads were aligned to the hg19 reference genome with the BWA-MEM algorithm [34]. The identification of SNPs and indels and genotyping were performed across all samples simultaneously using standard hard filtering parameters or variant quality score recalibration according to GATK Best Practices recommendations [35].WGS was performed with a minimum, median coverage of $30 X$.

\section{Immune response gene expression data}

Cancer tissues with immune response gene expression profile data were obtained from 99 colorectal cancer patients. RNA was prepared from formalin-fixed paraffinembedded (FFPE) tissue that was extracted with the RecoverAll Total Nucleic Acid Isolation Kit (Thermo Fisher Scientific). RNA concentration was determined on an Invitrogen ${ }^{\mathrm{TM}}$ Qubit $^{\mathrm{TM}}$ Fluorometer with the Qubit ${ }^{\mathrm{TM}}$ RNA High Sensitivity Assay (Thermo Fisher Scientific). Twenty nanograms of RNA was used for each reverse transcription reaction, and cDNA was prepared with the SuperScript $^{\mathrm{TM}}$ IV VILO ${ }^{\mathrm{TM}}$ Master Mix Kit. Immune response libraries were prepared using the Ion AmpliSeq ${ }^{\mathrm{Tm}}$ Kit for Chef DL8 with the Ion Chef ${ }^{\mathrm{m}}$ System and according to instructions in the Oncomine ${ }^{\mathrm{TM}}$ Immune Response Research Assay user guide (Pub. No. MAN0015867). The raw gene expression data were preprocessed using Torrent Suite (Thermo Fisher Scientific) and normalized with the min-max feature scaling approach.

\section{Statistical analysis}

The chi-square test and Fisher's exact test were used to assess the differences between groups. Kaplan-Meier curves were used to evaluate RFS, which was defined as the time between surgery and cancer recurrence. A $p$ value $<0.05$ was considered statistically significant.

\section{Machine learning model and analysis Detecting DSVs and data preprocessing}

We detected germline DSVs in cancer and non-cancer subjects simultaneously with PopDel from wholegenome DNA sequencing data [10]. DSVs were then filtered by the minor allele frequency (MAF). A MAF greater than or equal to 0.05 and occurring in at least $1 \%$ of the sample in each population was subjected to further analysis.

\section{Selecting DSVs for the cancer risk and immune expression correlation model}

We designed an attention-weighted model [36] to select important DSVs (Fig. 2a). This model is a MLP model based on the attention mechanism. During the learning process, the model automatically adjusts the weight of every DSV. The main aim of this model is to predict 
subjects with or without cancer. We used the deletion vector for each sample as the input of the attentionweighted model and then adopted binary cross-entropy as a loss function. After training the model, we obtained the weight of each DSV. We then selected cancer riskassociated DSVs with positive weights, which are important when classifying cancer and non-cancer samples. We correlated cancer risk-associated DSVs and immune gene expression data from 99 colorectal cancer patients. The gene expression data were normalized. An immune expression correlation table was established with the point-biserial correlation [37], which was used to correlate continuous variables with dichotomous variables, to determine the relationship between DSVs and immune gene expression.

\section{Prognostic candidate genes and survival stratification}

There are many survival analyses using the machine learning approach to achieve predicted results, especially survival-SVM [17] which can have better results. We can also know the importance of each DSVs to the model, and it can also be more interpretable. We selected prognosis-associated candidate DSVs by using the survival-SVM [17], which is the approach that can be used to predict the event time duration based on a given set of features. Therefore, we do feature selection base on the survival-SVM, which can select the most predictive prognosis associated with DSVs in the model. The candidate DSVs were clustered into two groups: the recurrence-associated DSV group and the nonrecurrence-associated DSV group. We measured the hazard ratio (HR) of each candidate deletion using Cox's proportional hazards model, which represents the probability of recurrence by giving the survival time of patients. We determined that DSVs with a positive log (hazard ratio) were recurrence-associated deletions, while DSVs with a negative log were non-recurrenceassociated deletions. The prognostic DSVs were selected with statistical significance in the hazard model. We used the Kaplan-Meier method for the survival analysis to compare the differences between the two survival curves using the log-rank test [38].

\section{Supplementary Information}

The online version contains supplementary material available at https://doi. org/10.1186/s40246-020-00302-3.

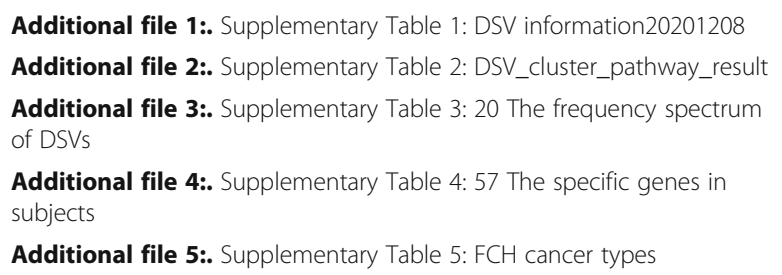

Additional file 6:. Supplementary Table 6: Cox proportional

Additional file 7:. Supplementary Table 7: 57 Six functional categories

Additional file 8:. Supplementary figures

\section{Acknowledgements}

The authors gratefully acknowledge the significant contribution of LY Hung for the helpful critiques and suggestions. We also acknowledge the essential work of the Taiwan Biobank in collecting the population-based genome data.

\section{Provenance and peer review}

Not commissioned; externally peer reviewed.

\section{Authors' contributions}

The conception and study design were done by PL, HC, and JC. The development of methodology was done by $\mathrm{PL}, \mathrm{CL}$, and JC. The acquisition of data was done by PL, YY, and MS. The statistical and computational analyses were done by $\mathrm{PL}, \mathrm{HC}, \mathrm{CL}$, and JC. The writing, review, and/or revision of the manuscript were done by $\mathrm{PL}, \mathrm{HC}, \mathrm{YY}, \mathrm{MS}$, and JC. The study supervision was done by JC. The authors have read and approved the final manuscript. All authors agree for publication

\section{Funding}

This work was supported in part by the Ministry of Science and Technology (MOST), Taiwan under Research Grant of MOST-108-2634-F-006-006 and MOST 108-2634-F-006-011 and Ministry of Health and Welfare (MOHW108TDU-B-211-124018 and MOHW108-TDU-B-211-133003). All authors have read and approved the manuscript

\section{Availability of data and materials}

The authors confirm that the data supporting the findings of this study are available within the article and its supplementary materials.

\section{Ethics approval and consent to participate}

This study was approved by the institutional review board of National Cheng Kung University Hospital (NCKUH) (A-ER-103-395 and A-ER-104-153) and conducted in accordance with the Declaration of Helsinki. All participants provided written informed consent.

\section{Consent for publication}

Not applicable

\section{Competing interests}

The authors declare that they have no competing interests.

\section{Author details}

${ }^{1}$ Department of Computer Science and Information Engineering, College of Electrical Engineering and Computer Science, National Cheng Kung University, Tainan, Taiwan. ${ }^{2}$ Institute of Medical Informatics, National Cheng Kung University, Tainan, Taiwan. ${ }^{3}$ Department of Oncology, National Cheng Kung University Hospital, College of Medicine, National Cheng Kung University, Tainan, Taiwan. ${ }^{4}$ Department of Internal Medicine, National Cheng Kung University Hospital, College of Medicine, National Cheng Kung University, Tainan, Taiwan. ${ }^{5}$ Graduate Institute of Clinical Medicine, College of Medicine, National Cheng Kung University, Tainan, Taiwan. ${ }^{6}$ Department of Obstetrics and Gynecology, National Cheng Kung University Hospital, College of Medicine, National Cheng Kung University, Tainan, Taiwan. ${ }^{7}$ Department of Pharmacology, National Cheng Kung University Hospital, College of Medicine, National Cheng Kung University, Tainan, Taiwan.

Received: 17 September 2020 Accepted: 22 December 2020

Published online: 11 January 2021

\section{References}

1. Escala-Garcia M, Guo Q, Dörk T, Canisius S, Keeman R, Dennis J, et al. Genome-wide association study of germline variants and breast cancerspecific mortality. Br J Cancer. 2019;120:647-57.

2. Tenesa A, Dunlop MG. New insights into the aetiology of colorectal cancer from genome-wide association studies. Nat Rev Genet. 2009;10:353-8. 
3. Sanchis-Juan A, Stephens J, French CE, Gleadall N, Mégy K, Penkett C, et al Complex structural variants in Mendelian disorders: identification and breakpoint resolution using short- and long-read genome sequencing. Genome Med. 2018:10:1-10.

4. Merker JD, Wenger AM, Sneddon T, Grove M, Zappala Z, Fresard L, et al. Long-read genome sequencing identifies causal structural variation in a Mendelian disease. Genet Med. 2018;20:159-63.

5. Thibodeau ML, O'Neill K, Dixon K, Reisle C, Mungall KL, Krzywinski M, et al. Improved structural variant interpretation for hereditary cancer susceptibility using long-read sequencing. Genet Med. 2020;22:1892-7.

6. Wang X, Oldani MJ, Zhao X, Huang X, Qian D. A review of cancer risk prediction models with genetic variants. Cancer Inform. 2014;13:19-28,

7. Kim BJ, Kim SH. Prediction of inherited genomic susceptibility to 20 common cancer types by a supervised machine-learning method. Proc Natl Acad Sci U S A. 2018;115:1322-7.

8. Eraslan G, Avsec Ž, Gagneur J, Theis FJ. Deep learning: new computational modelling techniques for genomics. Nat Rev Genet. 2019;20:389-403.

9. Cruz JA, Wishart DS. Applications of machine learning in cancer prediction and prognosis. Cancer Inform. 2006;2:59-77.

10. Kehr B, Helgadottir A, Melsted $P$, Jonsson $H$, Helgason $H$, Jonasdottir A, et al. Diversity in non-repetitive human sequences not found in the reference genome. Nat Genet. 2017;49:588-93.

11. Audano PA, Sulovari A, Graves-Lindsay TA, Cantsilieris S, Sorensen M, Welch AME, et al. Characterizing the major structural variant alleles of the human genome. Cell. 2019;176:663-675.e19.

12. Duncan L, Shen H, Gelaye B, Meijsen J, Ressler K, Feldman M, et al. Analysis of polygenic risk score usage and performance in diverse human populations. Nat Commun. 2019;10:1-9.

13. Guo X, Shi J, Cai Q, Shu XO, He J, Wen W, et al. Use of deep whole-genome sequencing data to identify structure risk variants in breast cancer susceptibility genes. Hum Mol Genet. 2018;27:853-9.

14. Jassal B, Matthews L, Viteri G, Gong C, Lorente P, Fabregat A, et al. The reactome pathway knowledgebase. Nucleic Acids Res. 2020;48:D498-503.

15. Zhang J, Walsh MF, Wu G, Edmonson MN, Gruber TA, Easton J, et al. Germline mutations in predisposition genes in pediatric cancer. N Engl J Med. 2015;373:2336-46.

16. Hanahan D, Weinberg RA. Hallmarks of cancer: the next generation. Cell. 2011:144:646-74.

17. Pölsterl S, Navab N, Katouzian A. Fast training of support vector machines for survival analysis. In: Lecture Notes in Computer Science (including subseries Lecture Notes in Artificial Intelligence and Lecture Notes in Bioinformatics). 2015. p. 243-59.

18. Binnewies M, Roberts EW, Kersten K, Chan V, Fearon DF, Merad M, et al. Understanding the tumor immune microenvironment (TIME) for effective therapy. Nat Med. 2018;24:541-50.

19. Fanipakdel A, Seilanian Toussi M, Rezazadeh F, Mohamadian Roshan N, Javadinia SA. Overexpression of cancer-testis antigen melanoma-associated antigen A1 in lung cancer: a novel biomarker for prognosis, and a possible target for immunotherapy. J Cell Physiol. 2019;234:12080-6.

20. Mao Y, Tang Q, Fan W, Tang X, Xu L, Zhu J, et al. A novel MAGE-A1-IgG antibody for lung adenocarcinoma. J Clin Oncol. 2017;35(15_suppl):e20085.

21. Franceschini A, Szklarczyk D, Frankild S, Kuhn $M$, Simonovic $\bar{M}$, Roth $A$, et al. STRING V9.1: Protein-protein interaction networks, with increased coverage and integration. Nucleic Acids Res. 2013;41:D808-15.

22. Heinze-Deml C, Maathuis MH, Meinshausen N. Causal structure learning. Annu Rev Stat Its Appl. 2018;5:371-91.

23. Spirtes P, Glymour C, Scheines R. Causation, prediction, and search, 2nd edition. 2000.

24. Pidugu VK, Wu MM, Yen AH, Pidugu HB, Chang KW, Liu CJ, et al. IFIT1 and IFIT3 promote oral squamous cell carcinoma metastasis and contribute to the anti-tumor effect of gefitinib via enhancing p-EGFR recycling. Oncogene. 2019;38:3232-47.

25. Chaturvedi $P$, Singh AP, Batra SK. Structure, evolution, and biology of the MUC4 mucin. FASEB J. 2008;22:966-81.

26. Oshimori N, Li X, Ohsugi M, Yamamoto T. Cep72 regulates the localization of key centrosomal proteins and proper bipolar spindle formation. EMBO J. 2009;28:2066-76.

27. Soh KP, Szczurek E, Sakoparnig T, Beerenwinkel N. Predicting cancer type from tumour DNA signatures. Genome Med. 2017;9:1-11.
28. Li Z, Qian R, Zhang J, Shi X. MiR-218-5p targets LHFPL3 to regulate proliferation, migration, and epithelial-mesenchymal transitions of human glioma cells. Biosci Rep. 2019;39:1-14.

29. Liang YK, Lin HY, Chen CF, Zeng D. Prognostic values of distinct CBX family members in breast cancer. Oncotarget. 2017;8:92375-87.

30. Ashburner M, Ball CA, Blake JA, Botstein D, Butler H, Cherry JM, et al. Gene ontology: tool for the unification of biology. Nat Genet. 2000;25:25-9.

31. Yu H, Kortylewski M, Pardoll D. Crosstalk between cancer and immune cells: Role of STAT3 in the tumour microenvironment. Nat Rev Immunol. 2007;7: $41-51$.

32. Kondou R, lizuka A, Nonomura C, Miyata H, Ashizawa T, Nagashima T, et al. Classification of tumor microenvironment immune types based on immune response-associated gene expression. Int J Oncol. 2019;54:219-28.

33. Chen $\mathrm{CH}$, Yang JH, Chiang CWK, Hsiung CN, Wu PE, Chang LC, et al. Population structure of Han Chinese in the modern Taiwanese population based on 10,000 participants in the Taiwan Biobank project. Hum Mol Genet. 2016;25:5321-31.

34. Li H. Aligning sequence reads, clone sequences and assembly contigs with BWA-MEM. Preprint at https://arxiv.org/abs/1303.3997(2013).

35. Depristo MA, Banks E, Poplin R, Garimella KV, Maguire JR, Hartl C, et al. A framework for variation discovery and genotyping using next-generation DNA sequencing data. Nat Genet. 2011:43:491-501.

36. Vaswani A, Shazeer N, Parmar N, Uszkoreit J, Jones L, Gomez AN, et al. Attention is all you need. Adv Neural Inf Process Syst. 2017;30:5999-6009.

37. Kornbrot D. Point biserial correlation. In: Wiley StatsRef: Statistics Reference Online. 2014.

38. Bewick V, Cheek L, Ball J. Statistics review 12: Survival analysis. Crit Care. 2004;8:389-94.

\section{Publisher's Note}

Springer Nature remains neutral with regard to jurisdictional claims in published maps and institutional affiliations.

Ready to submit your research? Choose BMC and benefit from:

- fast, convenient online submission

- thorough peer review by experienced researchers in your field

- rapid publication on acceptance

- support for research data, including large and complex data types

- gold Open Access which fosters wider collaboration and increased citations

- maximum visibility for your research: over $100 \mathrm{M}$ website views per year

At BMC, research is always in progress.

Learn more biomedcentral.com/submissions 